\title{
Minocycline-EDTA lock reduces bacteremia in dialysis catheters
}

An antibiotic lock solution

containing minocycline and ethylenediaminetetraacetic acid (EDTA) reduces catheter-related bacteremia in patients on hemodialysis, according to a randomized, controlled trial conducted at three centers in Brazil.

Infection is one of the two main complications associated with use of hemodialysis catheters, the other being dysfunction. Catheter-related bacteremia (CRB) is the most serious type of infection, and causes considerable morbidity and mortality in patients on dialysis. A number of studies have found that using antibiotic or antimicrobial substances combined with an anticoagulant reduces the risk of CRB compared with use of standard heparin lock solutions. No consensus has been reached on the use of such solutions, however, owing to concerns that use of antibiotic lock solutions may promote the emergence of resistant bacteria. "We reasoned that it would be rational to use an antibiotic in catheter locks that is not indicated for the treatment of systemic infections," state Campos and colleagues, authors of the latest paper. "Minocycline in combination with EDTA (M-EDTA) is highly effective in eradicating Staphylococcus epidermidis, Staphylococcus aureus and Candida albicans when these microorganisms are embedded in the biofilm covering catheter surfaces." An earlier study did not show a reduction in CRB with M-EDTA, but was underpowered. Another small randomized study showed that M-EDTA reduced CRB compared with heparin in tunneled

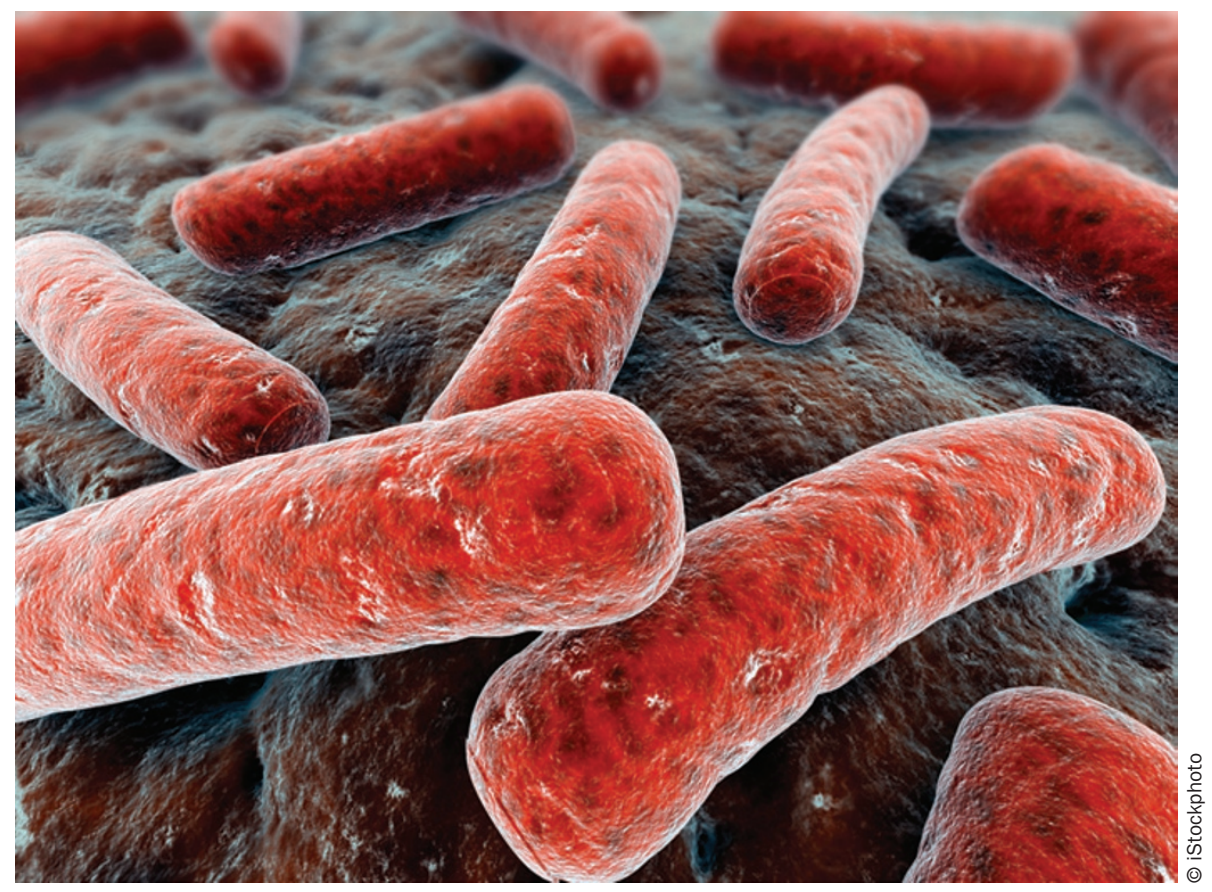

catheters. These findings led Campos et al. to initiate their randomized, controlled study comparing M-EDTA and heparin as catheter lock solutions.

The study included 204 incident tunneled and nontunneled catheters (in 150 patients); 17 of these were excluded and so the researchers analyzed results from 95 catheters filled with unfractionated sodium heparin and 92 catheters filled with M-EDTA solution. Over 90 days, CRB rates were significantly higher in the heparin group than in the M-EDTA group (4.3 per 1,000 catheter days versus 1.1 per 1,000 catheter days; $P=0.005)$. In addition, 90-day CRB-free survival was higher in the M-EDTA group than in the heparin group $(91.3 \%$ versus $69.3 \% ; P=0.005)$. Rates of catheter removal for dysfunction were similar in the two groups. "It is important to point out that a reduction in episodes of CRB implies less hospitalization, less cost, less morbidity and less mortality," note the authors.

\section{Rebecca Ireland}

Original article Campos, R. P. et al. Minocycline-EDTA lock solution prevents catheter-related bacteremia in hemodialysis. J. Am. Soc. Nephrol. http://dx.doi.org/ 10.1681/ASN.2010121306 